\title{
Work-Life Integration in Academia: From Myth to Reality
}

\author{
Sophie J. Barbu, Karen McDonald, Binnie Singh, and Laura Grindstaff
}

\begin{abstract}
Work-life integration is often considered the stuff of myth, especially for women in academia. The inherent conflict between an identity as a mother or parent and that as a working professional effectively limits diversity efforts in STEM. Addressing this conflict is therefore crucial to creating a more inclusive academic environment. Work-life integration has two fundamental components-structural and cultural. Workplace polices need to enable attainment of work and life goals; at the same time, the work culture is important in assuring individuals take advantage of existing policies. In this chapter, we review several work-life integration interventions at UC Davis, including the Partner Opportunity Program and Capital Resource Network. We discuss the challenges associated with these and other efforts during the implementation of our ADVANCE programs. We also make recommendations for improving work-life integration in academia and beyond - to turn myth into reality.
\end{abstract}

Keywords Work-life integration $\cdot \mathrm{STEM} \cdot$ Academia $\cdot$ Gender equality Childcare $\cdot$ Dual careers $\cdot$ Partner opportunity program

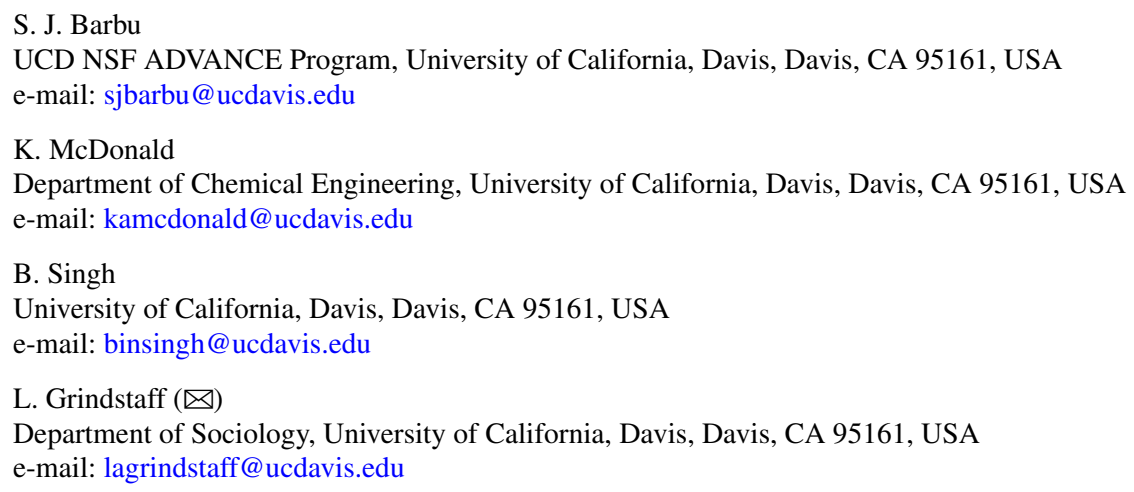




\section{Introduction}

In 2008, the Michelle R. Clayman Institute for Gender Research at Stanford University reported that $72 \%$ of academic faculty are in dual career relationships, with approximately half of that number in dual academic career partnerships (Schiebinger et al., 2008). The single career couple model of a primary breadwinner and a primary homemaker no longer characterizes academia, especially for faculty. The Clayman Institute report further linked achieving workforce diversity to the necessity of addressing dual career issues. An earlier analysis of the status of women at the Massachusetts Institute of Technology (MIT) concluded much the same thing: "the profession is set up in such a way that men academics routinely have families, while women, given current rules, find it much more difficult" (Bailyn, 2003, p. 139). Many professional women are childless, which has been referred to as a "creeping nonchoice" (Hewlett, 2002).

The assumption that "gender equality" means treating women faculty as if they are male is inequitable on its face, particularly with respect to childrearing. Failure to accommodate work-life integration discourages women from pursuing STEM careers in academia, and is thereby a barrier to inclusion. Given the prevalence of dual career couples and the distribution of work-life responsibilities across both members of the couple, this is no longer an issue for women alone-men who wish to be engaged fathers face many of the same barriers (Stovell et al., 2017). Thus, addressing work-life integration is vital for all faculty members, regardless of gender, although women are still likely to benefit more because responsibility for domestic life still falls disproportionately on them.

Relatedly, "devotion to work" is a culturally-valued attribute disproportionately associated with men; inseparable from measures of achievement, it too makes establishing a workplace culture supportive of work-life integration quite challenging (Williams et al., 2016). In part this is because, historically, "dedication" to work became implicitly entwined with "better" work (Williams et al., 2016). This culture of work devotion - what is referred to in the chapter, 'Barriers to Inclusion: Social Roots and Current Concerns,' as the "ideal worker norm," was enabled by the prevalence of single-career couples along with the clear separation of work and life/family responsibilities by gender (male breadwinner, female homemaker). The fact that singlecareer couples are now relatively rare has not led to a readjustment of workplace culture. There is, in essence, a tug-of-war between co-parenting and dual careers, which women feel especially keenly: they can choose between being viewed as a good worker or as a good parent, but never as both (Williams et al., 2016). Workfamily conflict consumes cognitive energy, detracting from the positiveness of either experience. Thus, it's clearly beneficial to address this conflict and enable a cognitive focus on work, unhindered by guilt about neglecting family, along with a cognitive focus on family, unhindered by guilt about neglecting work (Williams et al., 2016). Although the current culture of devotion to work is anachronistic, effecting meaningful change is difficult. Not only are work-life integration programs important in effecting change, individuals must be able to take advantage of those programs without worrying whether colleagues and coworkers will feel disappointed in them. In the interim, it is important to fully understand the countervailing pressures on 
performance versus parenthood, and to make work-life integration programs the new norm (Powell, 2019).

\section{The Status of Work-Life Integration Programs at UC Davis}

UC Davis supports integration of work and life across the career spectrum, although its policies are more generous for faculty compared to staff, lecturers, and postdoctoral scholars. Our Work Life Program was launched in 2003 and was awarded the World at Work 2017 Seal of Distinction. In 2006, UC Davis received a Faculty Flexibility Award through the ACE/Sloan Foundation, which provided funding to the program for leaves and reduced teaching loads for new parents, with the central administration covering the costs of replacement teaching. The program also enables extending the tenure clock, deferring merit actions, adjusting to part-time status temporarily as needed, supporting adoptive or foster children, and more. Either parent or both parents can take advantage of these policies. Our Work Life Program is unique in that it designates select faculty members to serve as Faculty Work-Life Advisors in almost every college and school on the campus. These advisors are trained to help those undergoing major family changes, particularly the birth, adoption, or foster placement of a child.

Although information about the program is readily available on the Academic Affairs website, it has been challenging to make faculty aware of its many benefits. This is partly because of normal turn-over in department leadership and staff support, as chairs or staff members in a position to share information about the program move out of their roles. Consequently, we continually seek avenues to get the word out, such as attending annual workshops held for faculty, including the New Faculty Workshop and the New Chairs Workshop (for newly appointed department chairs). We put together traveling "road shows"-presentations made to groups of chairs and department managers in each school or college on campus. Brochures about our program are provided to faculty candidates during the search process in the materials they receive about the campus. Finally, our work-life advisors approach faculty who they know are having or recently had a child to ensure they are aware of our programs.

\section{Overview of UC Davis Programs and Policies for Work-Life Integration for Faculty}

UC Davis Academic Affairs manages the training of program advisors-as mentioned above, these are trained faculty peers. They meet quarterly with a staff member in Academic Affairs to discuss new campus programs, share experiences with colleagues, and provide support to faculty considering starting families or 
needing support for them. This staff member also works directly with faculty, helping them understand and navigate the pertinent policies, answering questions, providing information on resources, and even advocating on their behalf with the leadership of a department or school or college.

\title{
3.1 Family Leave
}

In 2003, UC Davis began providing leave for faculty parents who had a child through either adoption or foster placement; the campus also provides central funding to cover replacement teaching costs. Since that time, women faculty members take advantage of family leave more often than male colleagues. Leave policies are based on the primary caregiver:

\begin{abstract}
Birthmother: One quarter/semester leave for a faculty woman with a single or multiple birth or a quarter/semester made of up a combination of six (6) weeks of leave and the remaining weeks as active service modified duties (ASMD). In the event of a summer birth/placement, the faculty mother may get three quarters/two semesters of ASMD, with complete relief of teaching for the fall quarter/semester, depending on the timing of the birth.

Non-birth mother: One quarter/semester of leave for the primary parent for adoption or foster placement. Two quarters/one semester of teaching relief/modified duties for the parent with $50 \%$ or more childcare responsibility. Faculty couples may receive special provisions.
\end{abstract}

\subsection{Extensions for Advancement Mandatory Timelines}

Assistant Professors or Senate Lecturers automatically receive a tenure clock extension for one year per each birth or child placement event, for a maximum of two extensions, for a total of two years. These extensions on the clock are noted automatically when the relevant university staff are made aware of the new child. UC policy has increased the allowable reasons for extensions on the clock to include a disability, a bereavement, or another significant life circumstance or event. Associate or Full Professors may also apply to postpone merits actions and/or promotions to accommodate childbirth, adoption, or foster or elder care. The length of postponement may not exceed one year per event, for a total of two years.

\subsection{Flexible Work}

Policies to accommodate flexible schedules also exist. Faculty may request to reduce their appointment to part-time for a finite period or permanently to deal with family needs. Faculty may also take advantage of other flexible work arrangements. Faculty 
schedules are conducive to flexibility. Besides teaching and fulfilling their other oncampus obligations, faculty may adapt their schedules appropriately and productively for scholarship; this can include working remotely.

\subsection{Childcare}

UC Davis has a wide variety of childcare options. Faculty can register for on-campus childcare, but receive no preferential treatment. All UC employees are eligible for Bright Horizons Care Advantage, with the following services for faculty (and staff):

- Sittercity, offering profiles, reviews, and background checks for prescreened caregivers, including babysitters, full- and part-time nannies, pet sitters, tutors, housekeepers, and individual senior caregivers

- Years Ahead, offering a nationwide network of certified senior care advisors, specialized facilities including memory and hospice care, independent and assisted living communities, and in-home healthcare and senior care companions

- Preferred enrollment at Bright Horizons childcare centers nationwide

- Tuition discounts at participating provider centers for ages two and older

- BrightStudy, tutoring and test preparation resources and referrals.

Employees have priority registration for children's summer camps through Campus Recreation and their respective unions. UC Davis also subsidizes on-campus childcare for student parents.

The UC Davis campus (logically, in Davis, California) currently has three childdevelopment centers, which are accredited by the National Association for the Education of Young Children (NAEYC); although convenient, there is no discount for faculty or staff. Currently, the UC Davis Health System (UCDHS) Sacramento campus, which includes UC Davis Medical Center, the UC Davis School of Medicine, the UC Davis Medical Group, and the Betty Irene Moore School of Nursing, is the sole health system within the University of California that still lacks campus-based childcare; this is a priority for future developments on that campus.

\subsection{Faculty Recruitment}

Family-friendly policies start with recruitment. In 2012, UC Davis implemented a family-friendly recruitment practice to make it easier for candidates who are parents of very young children to participate in on-campus interviews for faculty positions. This practice allows reimbursement of travel and hotel expenses for a person to accompany the prospective faculty mother or a single parent of either gender in order to breast- or bottle-feed a child under age two. Reimbursable hotel expenses may also include the costs associated with providing a crib in the hotel room. 


\subsection{Faculty Travel}

A newly revised travel policy, issued UC campus wide in July 2019, allows coverage for the travel costs of dependents of those employees who must travel for businessrelated reasons. This new policy increases the options for faculty to manage their family needs while simultaneously engaging in their scholarly activities. Each UC campus is currently devising implementation procedures for the policy.

\subsection{Dual Career Programs}

Partner Opportunity Program: One important aspect of faculty recruitment in today's academic world is candidates' frequent need to manage dual careers. The UC Davis Partner Opportunity Program (POP), one of the longest-running university dual career programs in the country, provides support to academic units in the recruitment and retention of outstanding faculty and in executive searches by assisting their partners and spouses in seeking employment at UC Davis. POP serves as a resource for candidates to explore their career goals, identify job opportunities and arrange contacts, access career counseling services through Human Resources, take advantage of training programs, and participate in informational interviews. It is important to note, however, that POP does not guarantee job placement.

It can be particularly challenging when both candidate spouses or partners are seeking faculty positions, because of the limited number available. POP can provide temporary bridging funds to support limited-term contract positions, so as to provide the partner of the main hire with more time to find longer-term employment. As a public institution, the university must adhere to mandatory open-search processes, which limits flexibility somewhat. Fortunately, the University of California as a whole and UC Davis in particular both include faculty hires as an acceptable reason for a search waiver, recognizing the challenges of creating a position specifically for an accompanying partner.

Capital Resource Network: Often, faculty being recruited have partners who are unable to find employment at UC Davis or who have a broader interest beyond the campus. The Capital Resource Network (CRN) was created in recent years to provide additional support to our newly hired faculty (or in fact any employee) or to help retain faculty. The CRN provides support early in the hiring process, when faculty candidates are considering accepting an offer. Candidates can meet with representatives of the $\mathrm{CRN}$ to discuss the services available for relocation and integration into the region (housing, schools, medical providers, special needs, and the like) and, if relevant, to get support for their partners' employment needs. The CRN team facilitates informed decision-making well in advance of an actual move if a candidate is hired. The $\mathrm{CRN}$ is a unique program within the UC system, and likely throughout the western region. 


\section{The Challenge of Childcare Provision}

Institutions of higher education throughout the United States increasingly find that high-quality childcare services are essential for recruiting and retaining faculty and staff (Boressoff, 2012). Because childcare is important throughout one's academic career, student and postdoctoral parents also need assistance to ensure their enrollment, retention, and graduation. Lack of adequate childcare, meaning the kind that is both available and affordable, can drive prospective parents away from a career in academia.

One of the initiatives of the UC Davis ADVANCE program is the Social Science Research Initiative (SSRI), which studies the familial, socio-culture, and institutional factors that either facilitate or impede the inclusion and success of Latina scientists pursuing academic careers. SSRI's preliminary findings indicate that newly hired Latina faculty on our campus chose UC Davis over other options in part because of its suite of generous work-life integration policies. Faculty elsewhere interviewed by the SSRI team reported relying heavily on childcare during the work week, and benefited greatly from extended hours of daycare assistance during periods when their own workdays got extended because of grant or publication deadlines. Working in close proximity to day care centers also benefit faculty because it means driving shorter distances and spending less time commuting (Saldana et al., 2013).

\subsection{Turning Talk into Action}

UC Davis has two administrative advisory committees that partially overlap with the Work Life Program: The UC Davis Child and Family Care Administrative Advisory Committee (CFCAAC) and Status of Women at Davis Administrative Advisory Committee (SWADAAC), both composed of faculty, staff, and students. Their main purpose is to advise UC Davis leadership on gender and/or family issues affecting the larger campus community. CFCAAC, in particular, works to improve childcare and family care programs for employees and students. Despite all these efforts, provision of childcare remains a concern for many faculty at UC Davis. Faculty report greater dissatisfaction with work-life balance on our campus than at other nationally comparable institutions, with women being more dissatisfied than men (see COACHE Survey Advisory Committee Highlighted Results, 2016-2917, https://academica ffairs.ucdavis.edu/faculty-satisfaction-survey-reports-coache). Thus, although it is important to have committees tasked with advising leadership, such advice it is of little use unless put into action.

A key challenge here is the commitment of resources coupled with turnover in administrative leadership. Subsidizing childcare or eldercare for faculty, staff, and students is costly, and so, while the recommendations of advisory committees are often acknowledged, they are not always implemented. A strongly committed campus leadership that views work-life integration as a priority is essential for turning talk 
into action; consequently, turnover in leadership can derail momentum because new leaders may need to be educated about the issues or have different priorities. Turnover disrupts institutional memory and makes it difficult to gain traction on childcare (and other) issues.

\subsection{Risk and Liability as Decision-Drivers}

In 2017, ADVANCE was approached by a planning committee for the national Latinas Research Network Conference to see if UC Davis would consider hosting its conference the following year involving approximately 500 participants. The committee had one specific request: could UC Davis provide onsite childcare for conference participants who brought children? So we rushed to conduct research on campus logistics and capacity.

We had never provided childcare at ADVANCE events, nor had we even heard of other large events held at UC Davis that did so. We contacted local childcare facilities both on and off campus to see if they could provide childcare at or near the anticipated conference building. We also reached out to CFCAAC to learn if they had any experience with providing on-site care. CFCAAC reported that although they periodically received such inquiries, they felt unable to help because they saw the barriers to provision as prohibitive.

We found that the ultimate barrier to identifying a viable on-campus childcare option for conference participants was the potential risk of liability to the campus for having children in care on campus. According to the Director of the WorkLife and Wellness unit in Human Resources, there are numerous considerations to bringing babysitters on campus, including appropriateness of facilities, numbers and ages of children, hours of care provided, qualifications of childcare providers, liability, need for insurance, and the "overall risk" that would have to be vetted by the Risk Management Department. Unfortunately, all these factors combined created bureaucratic hurdles that could not be surmounted in time for the conference. Creating childcare programming as part of conference programming, although it would have greatly helped some participants, was deemed too labor- and cost-intensive in view of the limited number of children who would likely be served.

Risk and liability are important considerations, of course, but they should not block action or prevent workable solutions. Complicated issues will never be resolved if we decide nothing can be done because they are too complicated. Clearly, and especially with regard to childcare, complete inaction minimizes liability to zero. 


\section{5 (Lack of) Childcare as a Barrier to Inclusion in STEM}

Many academics (disproportionately women) rightly perceive that work-life integration is no easy feat because the culture of overwork and the desire to fulfil family responsibilities are in tension. The difficulty of balancing both can be visible to undergraduate as well as graduate students. One program on our campus that aims to reduce this tension is the Planned Educational Leave Program (PELP), which allows graduate students to take up to one year off (unpaid) for reasons related to illness, childbirth, childcare, eldercare, etc. and remain classified as students in good standing. However, this interruption can reduce access to support, including financial aid, academic employment, and the student health insurance program. Students who return from PELP status regain eligibility for these forms of support. Other options for student parents after childbirth or adoption include financial assistance, the Breastfeeding Support Program, the ability to enroll dependents in a voluntary healthcare plan, and eligibility for a Graduate Student Childcare Grant, which is not based on financial need. Graduate and professional students are also eligible to receive financial need-based childcare grants, which allow students to choose and schedule a childcare provider who works best for them.

Female students completing a PhD are more likely to enroll in PELP (19.3\%, compared to $15.5 \%$ of male students); hence, median time-to-degree (TTD) for women is longer (5.7 years compared to 5.5 years for men). TTD excluding quarters in PELP is 5.5 years for both women and men. There is no difference by sex, race, or ethnicity in TTD among master's students who use PELP, nor is there a difference by race or ethnicity among doctoral students. Students typically learn about flexibility from their graduate advisors and graduate program staff.

\section{Lessons Learned}

\subsection{Communicate in Multiple Formats}

One issue that repeatedly stymied us was the prevailing, campus-wide lack of knowledge of our existing work-life integration policies and programs. Work-life advisors talked of having to identify new parents and then seek them out in order to provide appropriate advice. This suggested two things: (1) that in spite of advising new faculty and department chairs of the existence of these programs, the information was quickly forgotten, and (2) that faculty were likely finding advice elsewhere. In discussions with faculty, many referred to informal parenting networks that provided not merely moral support but also childcare backup as well as detailed information about childcare options. We concluded that institutions should embrace new-parent networks, provide cyberspace for their development, and work to ensure networks' awareness of campus policy and programs. In addition, electronic mailing listservs for faculty could be created to serve this same purpose. 


\subsection{Understand the Link Between Work-Life Integration and Diversity}

The ideal worker norm - the idea that unencumbered dedication to work is an essential, if not the essential, component of a good worker (in the case of academics, one who achieves "superior intellectual attainment")—is a barrier to inclusion. The conflict between one's work identity and one's family identity often leads faculty to minimize or hide the family side of the equation. For example, women report more frequently than men that they avoid displaying photos of children on desks because it may communicate that they are insufficiently devoted to research, teaching, and service. The ideal worker norm creates a culture of exclusion for parents and may discourage the pursuit of academic careers for both women and men.

\subsection{Do Not Take "It's Too Complicated..." for an Answer}

In discussions of enhanced work-life integration programs, specifically in the provision of childcare as a work benefit, we often heard the excuse "it's too complicated" as a reason for not solving the central issue of affordable, available, and appropriate childcare. Our campus needs child-friendly spaces where faculty parents can collaborate with administrators to create solutions to childcare issues-by establishing a parents' cooperative on leased space, for example. But such ideas are shot down because of assumed liability issues.

UC Davis is not alone in struggling to address this problem in academia, and if liability is one obstacle, money is another. A recent article about childcare issues at Oregon State University cites a U. S. Department of Health and Human Services recommendation that childcare should not exceed $10 \%$ of total family income (Hogue, 2018). The average cost of childcare in California currently is approximately $\$ 1000$ per month, or $\$ 12,000$ per year, with variations by county and type of care (Kidsdsta, 2019).

Using the $10 \%$ rubric, a family would need a minimum income of $\$ 120,000$ for one child, or $\$ 240,000$ for two children. The UC systemwide salary scales (effective July 1, 2019) are well below these values for junior faculty. The salary range for assistant professors varies by unit and type of appointment. For academic year or nine-month appointments, the salary range is from $\$ 60,000$ (step I) to $\$ 78,900$ (step VI). Yearly childcare costs for a single child would range from 20 to $15 \%$ of salary. For 12-month or fiscal year appointees the salary range is $\$ 70,000$ to $\$ 91,600$, or from 17 to $13 \%$ of salary. Health science faculty are on a higher scale, with salary ranging from $\$ 72,800$ to $\$ 95,300$ at the ranks of assistant professor, or $16-12 \%$ of salary for childcare for a single child. Faculty in each of these groups raised concerns about the cost of childcare. Interestingly, faculty in the College of Engineering reported that the cost of childcare was not an issue. The Engineering faculty salary scale for assistant professors ranges from $\$ 93,200$ to $\$ 117,100$, or from 13 to $10 \%$ of the 
average cost of childcare for a single child. This suggests that a viable alternative to providing childcare as a work benefit is the adjustment of salary scales along the recommendation that 10 percent of total income be devoted to childcare. Thus, rather than just accepting "no" with respect to childcare, we also need to consider viable, low-liability alternatives such as linking salary scales to the average cost of childcare for a single child. This would also address another issue we heard frequently-that childcare was not an "equitable benefit" because some faculty are not parents or are parents with older children and therefore ineligible for the benefit. Increasing salary scales for all rather than implementing a benefit would solve this problem.

\subsection{Create a Sustained Commitment for Action}

The final lesson we learned is that addressing work-life integration requires a sustained commitment for action on the part of administrators as well as faculty and that the commitment has to be visibly promoted. Through our work on ADVANCE, we contacted the two campus entities mentioned earlier: CFCAAC (Child and Family Care Administrative Advisory Committee) and SWADAAC (Status of Women at Davis Administrative Advisory Committee). From these committees we learned that faculty in the Health System (housed at the university's Sacramento campus) believed lack of childcare to be a major workplace problem, yet repeated efforts to address it had failed. When we took the issue to the executive leadership, we found there was overwhelming support to address childcare issues and to establish a childcare facility on the Sacramento campus. For various reasons, the Health System faculty on the Sacramento campus and the administrative leadership on the main UCD campus were not in conversation. With the support of the Chancellor, SWADAAC got direct access to the Planning Director of a new campus development in Sacramento called "Aggie Square." The committee successfully made the case for creating a new child care center near the UC Davis Health campus. To address a shortage of available licensed childcare for local working families, UC Davis Health, SMUD and Sacramento State University joined forces to create a new child care center in Sacramento, CA.

This incident reveals how important it is to involve in decision-making those standing committees or permanent advisory groups that advocate on behalf of diversity issues so they can keep such issues at the forefront of planning and development. A key challenge here is the sheer volume of campus groups and committees and the low-profile given to work-life integration. Work-life integration efforts are critical to faculty recruitment and retention; another recommendation, therefore, to ensure the visibility and centrality of those programs and committees that undertake such efforts.

Our final recommendation is to make work-life integration a priority for funding. As we have seen, childcare is expensive, especially in California, and whatever 
strategies are proposed to help faculty parents balance commitments to work and home will require substantial investment. Whether by increasing salaries, providing childcare stipends or reimbursements, providing affordable childcare on sight, or coordinating childcare at a local facility, funding is critical because all options are costly. Only a broad-based, collective commitment that runs from the top to the bottom of the academic hierarchy will make it happen.

\section{Conclusion}

Work-life conflict leads to career and job dissatisfaction. Our acceptance of the importance of work devotion as a metric of intellectual attainment permeates all aspects of academic life. Individuals who place the quest for knowledge ahead of family are perceived as highly dedicated and committed to advancing society. This attitude, still widely held, raises barriers to inclusion for those faculty equally committed to being engaged parents. If we want to turn the myth of work-life integration into reality, we must not only change the policies and practices of academic institutions, we must also address the cultural norms and values that underwrite them.

\section{References}

Bailyn, L. (2003). Academic careers and gender equity: Lessons learned from MIT. Gender, Work and Organization, 10, 137-153.

Boressoff, T. (2012). Varieties of campus child care toolkit. Institute for Women Policy Research.

Hewlett, S. A. (2002). Executive women and the myth of having it all. Harvard Business Review, 2002: April. https://hbr.org/2002/04/executive-women-and-the-myth-of-having-it-all.

Hogue, T. (2018). Faculty, staff, struggle to find affordable, accessible child care. Available online at https://today.oregonstate.edu/story/faculty-staff-struggle-find-affordable-accessible-childcare

KidsData. (2019). Data available from website: https://www.kidsdata.org/topic/1849/ child-care-cost/table\#fmt=2358\&loc $=2,127,347,1763,331,348,336,171,321,345,357,332$

,324,369,358,362,360,337,327,364,356,217,353,328,354,323,352,320,339,334,365,343,330 $, 367,344,355,366,368,265,349,361,4,273,59,370,326,333,322,341,338,350,342,329,325,359$ $, 351,363,340,335 \& \mathrm{tf}=88 \& \mathrm{ch}=984,985,222,223 \&$ sortColumnId=0 \&sortType $=$ asc

Powell, K. (2019). Babies on board: Why US scientist-mums need support in the early years of parenthood. Nature, 569, 149-151.

Saldana, L. P., Castro-Villarreal, F., \& Sosa, E. (2013). "Testimonios" of Latina junior faculty: Bridging academia, family, and community lives in the academy. Educational Foundations, 27, $31-48$.

Schiebinger, L., Davies Henderson, A. R., \& Gilmartin, S. K. (2008). Dual career academic couples: What universities need to know. Retrieved from Stanford University, Michelle R. Clayman Institute for Gender Research. https://gender.stanford.edu/publications/dual-career-aca demic-couples-what-universities-need-know

Stovell, C., Collinson, D., Gatrell, C., \& Radcliff, L. (2017). Rethinking work-life balance and wellbeing: The perspectives of fathers. In S. C. L. Cooper \& M. Leitner (Eds.), The Routledge companion to wellbeing at work (pp. 221-234). Routledge.

Williams, J. C., Berdahl, J. L., \& Vandello, J. A. (2016). Beyond work-life "integration." Annual Review of Psychology, 67, 515-539. 
Open Access This chapter is licensed under the terms of the Creative Commons Attribution 4.0 International License (http://creativecommons.org/licenses/by/4.0/), which permits use, sharing, adaptation, distribution and reproduction in any medium or format, as long as you give appropriate credit to the original author(s) and the source, provide a link to the Creative Commons license and indicate if changes were made.

The images or other third party material in this chapter are included in the chapter's Creative Commons license, unless indicated otherwise in a credit line to the material. If material is not included in the chapter's Creative Commons license and your intended use is not permitted by statutory regulation or exceeds the permitted use, you will need to obtain permission directly from the copyright holder.

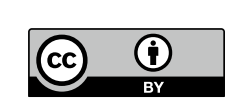

